\title{
A Vectored Measles Virus Induces Hepatitis B Surface Antigen Antibodies While Protecting Macaques against Measles Virus Challenge
}

\author{
Jorge Reyes del Valle, ${ }^{1}$ Patricia Devaux,${ }^{1}$ Gregory Hodge, ${ }^{2}$ Nicholas J. Wegner, ${ }^{1}$ \\ Michael B. McChesney, ${ }^{2}$ and Roberto Cattaneo ${ }^{1 *}$ \\ Molecular Medicine Program and Virology and Gene Therapy Graduate Track, Mayo Clinic College of Medicine, Rochester, \\ Minnesota 55905, ${ }^{1}$ and California National Primate Research Center and Department of Pathology and \\ Laboratory Medicine, School of Medicine, University of California-Davis, Davis, California $95616^{2}$
}

Received 30 April 2007/Accepted 11 July 2007

\begin{abstract}
Hepatitis B virus (HBV) acute and chronic infections remain a major worldwide health problem. Towards developing an anti-HBV vaccine with single-dose scheme potential, we engineered infectious measles virus (MV) genomic cDNAs with a vaccine strain background and expression vector properties. Hepatitis B surface antigen (HBsAg) expression cassettes were inserted into this cDNA and three MVs expressing HBsAg at different levels generated. All vectored MVs, which secrete HBsAg as subviral particles, elicited humoral responses in MV-susceptible genetically modified mice. However, small differences in HBsAg expression elicited vastly different HBsAg antibody levels. The two vectors inducing the highest HBsAg antibody levels were inoculated into rhesus monkeys (Macaca mulatta). After challenge with a pathogenic MV strain (Davis ${ }_{87}$ ), control naive monkeys showed a classic measles rash and high viral loads. In contrast, all monkeys immunized with vaccine or a control nonvectored recombinant vaccine or HBsAg-expressing vectored MV remained healthy, with low or undetectable viral loads. After a single vaccine dose, only the vector expressing HBsAg at the highest levels elicited protective levels of HBsAg antibodies in two of four animals. These observations reveal an expression threshold for efficient induction of HBsAg humoral immune responses. This threshold is lower in mice than in macaques. Implications for the development of divalent vaccines based on live attenuated viruses are discussed.
\end{abstract}

Vaccines based on live viruses can be highly effective and easy to produce and deliver. Smallpox was eradicated with live vaccinia virus, and a live attenuated poliovirus vaccine has been at the core of the polio eradication campaign (17). Worldwide measles virus (MV) vaccination prevents an estimated 80 million cases and 4.5 million deaths annually (45) with minimal severe adverse effects, on average less than 10 in 1 million doses (34). With the currently recommended vaccination scheme, the first dose of vaccine given at 10 to 12 months of age confers long-lasting immunity to $95 \%$ of vaccinees (16). The second dose, given to 6-year-olds, raises the conversion rate to nearly $100 \%$, eliminating primary vaccine failures (45). The two-dose strategy has been credited with elimination of indigenous measles in several countries (9), and the live attenuated MV vaccine is considered to be one of the safest and most cost-effective health tools available (28).

$\mathrm{MV}$ is a nonsegmented negative-strand RNA virus replicating in the cytoplasm. Vaccine safety and efficacy are sustained by lack of recombination, lack of a DNA replication phase, established vaccine production methods, and effective distribution networks $(2,50)$. A reverse genetic system (37) allows generation of recombinant MV expressing heterologous proteins, including those of other pathogens (48). For this, coding

\footnotetext{
* Corresponding author. Mailing address: Molecular Medicine Program, Mayo Clinic and Foundation, Guggenheim 18-42B, 200 First Street SW, Rochester, MN 55905. Phone: (507) 284-0171. Fax: (507) 262-2122. E-mail: cattaneo.roberto@mayo.edu.

${ }^{\nabla}$ Published ahead of print on 18 July 2007.
}

regions are inserted between duplicated MV-specific gene start and gene end motifs that direct transcription by the viral RNAdependent RNA polymerase. These expression cassettes are named additional transcription units (ATUs). The genomic location of an ATU determines the amount of protein expressed due to the sequential attenuation of transcription at gene ends $(6,18)$.

Work towards developing recombinant MV with additional vaccine specificities has begun: genes from hepatitis B virus (HBV) (43), simian and human immunodeficiency viruses (24, 52), mumps virus (50), and West Nile virus (10) have been inserted into different positions in the MV genome and thus expressed at different levels. The immunogenicities of vectored MVs and, in one case, their vaccine efficacies have been characterized in rodent and primate animal models. An MV-based candidate vaccine protected interferon (IFN) receptor-deficient mice against West Nile virus challenge (10).

The widely used HBV vaccine is based on Saccharomyces cerevisiae-expressed small surface antigen (hepatitis B surface antigen [HBsAg]) and has a three-dose schedule (22). This vaccine provides enduring and protective immunity, but compliance with this regimen is often low, and a long-desired global immunization is not in sight. To facilitate HBV eradication, alternatives have been proposed, including a two-dose scheme (1, 4). Replicating HBsAg-expressing viral vectors have also been generated: vaccinia virus $(27,44)$-, varicellazoster virus (20, 42)-, adenovirus (25)-, and MV-based vectors (43) produce HBsAg to high levels and elicit protective antiHBsAg antibodies in animal models. 
Here, we report the production of an MV cDNA with a vaccine background and of vectored MVs based on this cDNA but expressing HBsAg at different levels. Three of these vectored MVs replicated to high titers, and two induced high levels of HBsAg antibodies in mice. These two viruses were inoculated into rhesus monkeys (Macaca mulatta). Both retained full vaccine competence, protecting the hosts against challenge with a wild-type MV, but only one vector induced protective levels of HBsAg antibodies.

\section{MATERIALS AND METHODS}

Cells and viruses. Vero/hSLAM cells (31) and the helper 293-3-46 cell line (37) were maintained as monolayers in Dulbecco's modified Eagle's medium (Mediatech Inc., Herndon, VA) supplemented with $10 \%$ fetal calf serum, $1 \%$ penicillin-streptomycin (Mediatech), and 0.5 and $1.2 \mathrm{mg} / \mathrm{ml} \mathrm{G} 418$ (Mediatech), respectively.

Recombinant MVs were generated as described by Radecke et al. (37). Briefly, the helper cell line 293-3-46 stably expressing MV-N, MV-P, and T7 polymerase was transfected by calcium phosphate precipitation using the ProFection kit (Promega, Madison, WI) with two plasmids, one containing the relevant MV genome and the other coding for the MV polymerase (pEMCLa). Three days after transfection, the helper cells were overlaid on Vero/hSLAM cells, and the appearance of infectious centers was monitored. Then single syncytia were picked and propagated on Vero/hSLAM cells. To prepare virus stocks, Vero/ hSLAM cells were infected at a multiplicity of infection (MOI) of 0.03 and incubated at $32^{\circ} \mathrm{C}$. Cells were scraped in Opti-MEM (Gibco) and particles released by two freeze-thaw cycles.

Virus growth characteristics were determined by infecting Vero/hSLAM cell monolayers at a MOI of 0.03 and incubating them at $37^{\circ} \mathrm{C}$. Infected cells and supernatants were collected and lysed by one cycle of freeze and thaw $12,24,36$, 48,72 , and $96 \mathrm{~h}$ postinfection to determine virus titers by $50 \%$ end point dilution $\left(50 \%\right.$ tissue culture infectious dose $\left.\left[\mathrm{TCID}_{50}\right]\right)$ on Vero/hSLAM cells using the Spearman-Kärber method (21).

Plasmid construction. To correct three coding mutations in the polymerase (L) gene of the MV genome of $\mathrm{pB}(+) \mathrm{MVvac}(11)$, site-directed mutagenesis was performed using the QuikChange system (Stratagene, La Jolla, CA). Mutagenesis (T331I, E429D, and N1805S) was executed in a cassette vector covering the $\mathrm{L}$ gene sequence, pSK-Lvac (our unpublished data). After mutagenesis the corrected $\mathrm{L}$ gene was reinserted into a genomic plasmid named $\mathrm{pB}(+) \mathrm{MVvac} 2$. The complete coding identity of the plasmid sequence with those of both the Moraten and the Schwartz vaccine strains was confirmed (these two sequences are identical in spite of a nominally different origin, GenBank accession numbers AF266287 and AF266291) (32, 33).

For construction of the vectored MV, a previously described ATU $(37,43)$ containing the N/P intergenic region (TTATAAAAAACTTAGGAACCAGGT; the three intergenic nucleotides are underlined) was amplified by PCR and transferred to $\mathrm{pB}(+) \mathrm{MVvac2}$-based vectors. The original ATU was amplified either from plasmid $\mathrm{p}(+) \mathrm{MPrGFPV}$ or from plasmid $\mathrm{p}(+) \mathrm{MHrGFPV}(50)$ for subcloning in position $\mathrm{P}$ or $\mathrm{H}$, respectively. To facilitate future cloning of foreign genes and in compliance with the "rule of six", six nonoverlapping restriction sites (36 nucleotides) were inserted as specified in Fig. 1A. These ATUs were then inserted in $\mathrm{pB}(+) \mathrm{MVvac} 2$ in positions 3373, 9175, and 15786 of the MVvac2 genome (same numbers as in GenBank accession no. AF266287), giving rise to $\mathrm{pB}(+) \mathrm{MVvac} 2(\mathrm{ATU}) \mathrm{P}, \mathrm{pB}(+) \mathrm{MVvac} 2(\mathrm{ATU}) \mathrm{H}$, and $\mathrm{pB}(+) \mathrm{MVvac} 2$ (ATU)L, respectively.

The HBsAg coding sequence, obtained from $\mathrm{p}(+)$ MVHBs $(43)$ as a MluIBssHII restriction fragment, was then cloned into the corresponding sites of the ATUs of two $\mathrm{pB}(+) \mathrm{MVvac} 2(\mathrm{ATU})$ plasmids to obtain $\mathrm{pB}(+) \mathrm{MVvac} 2(\mathrm{HBs} \mathrm{Ag}) \mathrm{P}$ and $\mathrm{pB}(+) \mathrm{MVvac} 2(\mathrm{HBs} \mathrm{Ag}) \mathrm{H}$. The plasmid $\mathrm{pB}(+) \mathrm{MVvac} 2(\mathrm{HBs} \mathrm{Ag}) \mathrm{L}$ was obtained after transferring an MluI-AatII restriction fragment from $\mathrm{pB}(+) \mathrm{MVvac} 2$ (HBsAg)P as the donor of the HBsAg coding sequence. The integrity of the new junctions was verified by sequencing.

HBsAg expression analysis. For analysis of HBsAg expression Vero/hSLAM cells were seeded in a 35-mm-diameter six-well plate and infected with MVvac2 or its HBsAg-expressing derivates at a MOI of 0.5 . Twenty hours after infection the cells were methionine and cysteine starved for $15 \mathrm{~min}$ and labeled with 100 $\mu \mathrm{Ci} / \mathrm{ml}$ of Tran ${ }^{35} \mathrm{~S}$ label (ICN Pharmaceuticals, Irvine, CA) for $4 \mathrm{~h}$. The cells were lysed by incubation with radioimmunoprecipitation assay buffer $(50 \mathrm{mM}$ Tris [pH 7.5], $150 \mathrm{mM} \mathrm{NaCl}, 1 \% \mathrm{NP}-40,0.5 \%$ sodium deoxycholate, and $0.1 \%$ sodium dodecyl sulfate [SDS]) plus protease inhibitors (protease inhibitor cock- tail set I; Calbiochem, Darmstadt, Germany). The antigenic material was immunoprecipitated with protein A-agarose (Pierce, Rockford, IL) using $2.5 \mu \mathrm{l}$ of rabbit anti-MV $\mathrm{H}$ cytoplasmic tail polyclonal serum (5) or $2.5 \mu \mathrm{l}$ of rabbit anti-HBsAg H651 polyclonal antibody (a kind gift from Heinz Schaller, University of Heidelberg).

For flow cytometry analysis Vero/hSLAM cells were infected at a MOI of 2. To avoid syncytium formation, $0.2 \mathrm{mM}$ fusion inhibitor peptide Z-D-Phe-PheGly-OH (40) was added after the inoculum was removed. After $24 \mathrm{~h}$, cells were detached, fixed, permeabilized with $0.01 \%$ Triton X-100, and stained with 1:500 anti-MV $N$-fluorescein isothiocyanate (FITC) monoclonal antibody (Chemicon, Temecula, CA) or 1:100 anti-HBsAg mouse monoclonal antibody 3E7 (DakoCytomation, Carpinteria, CA). The reaction was developed by incubation with goat anti-mouse-FITC (Jackson Immunoresearch, West Grove, PA). Flow cytometry was performed using a BD-FACScalibur system (BD Biosciences, San Jose, CA) and analyzed using CellQuest software (BD Biosciences, San Jose, CA).

To quantitate HBsAg yields, $5 \times 10^{5}$ Vero/hSLAM cells were infected with the vectored MV at a MOI of 0.03 and supernatants were collected at 24, 48, and $72 \mathrm{~h}$ and precleared. HBsAg was detected by the HBsAg enzyme-linked immunosorbent assay (ELISA) Abazyme (Needham, MA) kit and quantified by a parallel line assay with a known standard (HBsAg ay, lot number 23090936; Chemicon, Temecula, CA).

HBsAg density determination. Supernatants of $7 \times 10^{6}$ infected cells were first clarified by centrifugation at $8,000 \mathrm{rpm}$ for $30 \mathrm{~min}$ in an SLA600TC rotor and then pelleted by ultracentrifugation at $35,000 \mathrm{rpm}$ for $18 \mathrm{~h}$ in an SW41Ti rotor. The pellet was resuspended in $1 \mathrm{ml}$ TNE buffer ( $1 \mathrm{mM}$ Tris [pH 7.8], $100 \mathrm{mM}$ $\mathrm{NaCl}, 10 \mathrm{mM}$ EDTA), brought to $20 \%$ sucrose in TNE, and then layered on top of a $20,30,40,50$, and $60 \%$ (2 ml each) sucrose-TNE step gradient, followed by ultracentrifugation in the same rotor at $29,000 \mathrm{rpm}$ for $16 \mathrm{~h}$. To determine the particle density and contents, 1-ml fractions were taken from the top and weighed. An aliquot of each fraction was analyzed by ELISA, and a $25-\mu$ l aliquot was subjected to $12.5 \%$ SDS-polyacrylamide gel electrophoresis (PAGE) and transferred to nitrocellulose for immunoblotting using a rabbit polyclonal antiMV-N at 1:30,000 dilution and a horseradish peroxidase (HRP)-conjugated secondary antibody. The reaction was developed using a chemiluminescence kit (SuperSignal West Femto maximum; Pierce, Rockford, IL). To detect HBsAg, we used a monoclonal antibody (anti-HBsAg-HRP) supplied as a secondary antibody in the HBsAg ELISA Abazyme kit and developed the reaction with the ECL-plus detection system (Amersham Biosciences Corp., Piscataway, NJ).

Mouse inoculation. All animal experimental procedures were performed according to a protocol previously approved by the Mayo Clinic Institutional Animal Care and Use Committee. The MV-susceptible mouse line Ifnar ${ }^{\text {ko }}$ CD46Ge (29) was used as a host. Ten mice per group were inoculated by the intraperitoneal route with $5 \times 10^{4} \mathrm{TCID}_{50}$ of either MVvac2 or its HBsAgexpressing derivatives. As a positive control a group of mice was inoculated with MVHBs, a previously reported HBsAg-expressing MV (43). Mice were bled at 14 and 28 days postinoculation, and serum was separated and stored at $-20^{\circ} \mathrm{C}$ until use.

Inoculation of rhesus macaques. MV-seronegative rhesus monkeys, housed at the California National Primate Research Center in accordance with the regulations of the Association for the Assessment and Accreditation of Laboratory Animal Care, were bled under ketamine sedation. Two control monkeys were vaccinated subcutaneously (s.c.) with $0.5 \mathrm{ml}$ of live attenuated MV (Attenuvax; Merck and Co., NJ). The titer of a vaccine dose is declared as greater than or equal to $10^{3} \mathrm{TCID}_{50}$, but we repeatedly measured about $10^{4}$ infectious units per dose. Therefore, $10^{4}$ infectious units of the vectored MV was also used for s.c. vaccination.

The monkeys were challenged by conjunctival/intranasal inoculation of $10^{5}$ infectious units of the Davis ${ }_{87}$ isolate of MV grown in Vero/hSLAM cells. The animals were monitored daily for anorexia, depression, coughing, diarrhea, and skin rash. They were bled on days $0,4,7,14$, and 28 postimmunization or postchallenge. Measles viremia was quantified by end point dilution coculture with Raji cells as described previously (36). Tissue culture infectious units were calculated by the method of Reed and Muench (39).

Characterization of the humoral immune response in mice and macaques. MV neutralizing antibody titers were determined in a plaque reduction assay by incubating serum dilutions (mouse or macaque serum) with 50 PFU of MVvac(GFP)N (our unpublished data) expressing green fluorescence protein and were expressed as $90 \%$ plaque reduction fluorescence-forming units. AntiHBsAg antibody titers were determined with a species-independent quantitative ELISA kit (Bioelisa anti-HBs; Biokit, Barcelona, Spain) and expressed as milliinternational units per milliliter by comparison with World Health Organization standards supplied by the manufacturer. 
A

$\mathrm{pB}(+) \mathrm{MVvac} 2$

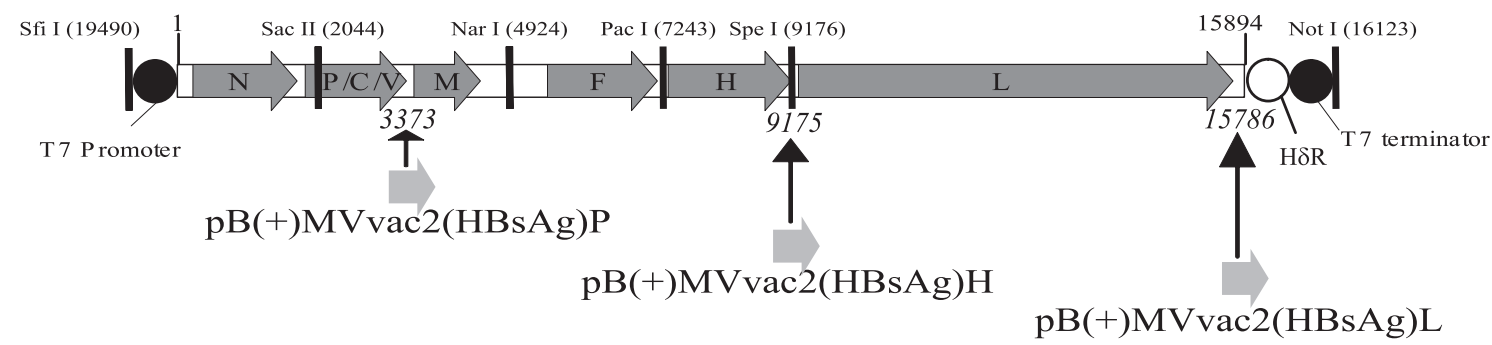

ATU (108nt)

MluI BsiWI NruI SnaBI BssHII AatII

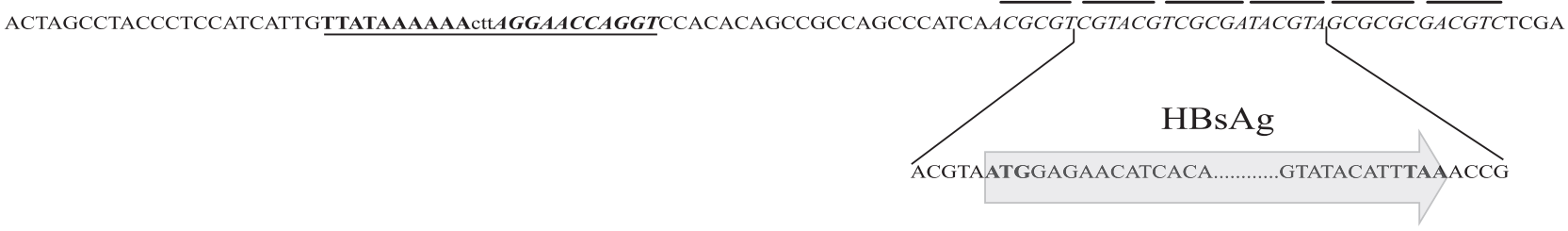

B
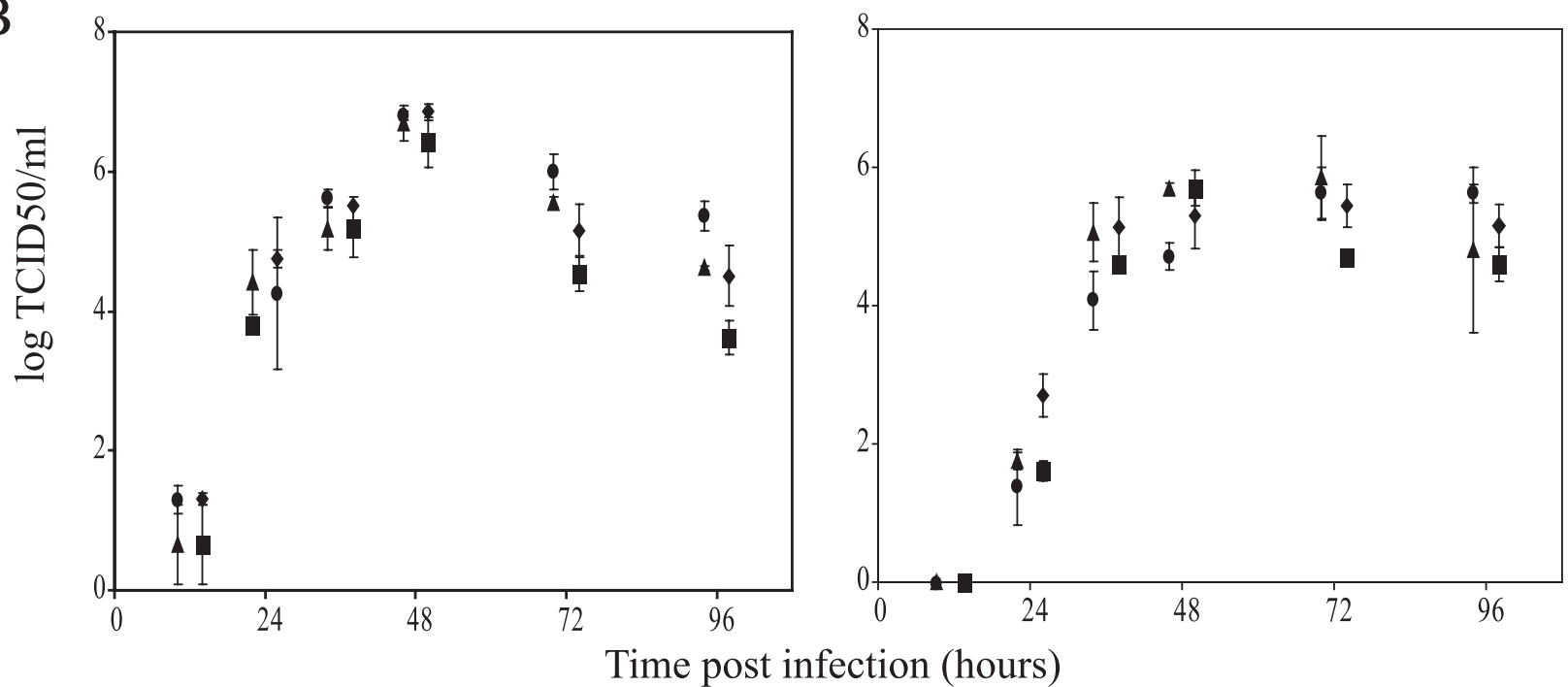

FIG. 1. Generation and characterization of vectored MV expressing HBsAg. (A, top) Map of pB(+)MVvac2, the plasmid containing an MV genome with vaccine-identical coding capacity and strategically located unique restriction sites. The coding regions of the MV genes are represented by arrowed dark gray boxes. The T7 promoter and terminator, as well as unique restriction sites, are indicated. H8R, hepatitis delta virus ribozyme. Names of the plasmids used to generate the vectored MVs that express HBsAg at different levels and the positions of the ATU inserted in these plasmids are shown below the $\mathrm{pB}(+) \mathrm{MVvac} 2$ map. (A, bottom) Nucleotide sequence of the ATU. The conserved termination (boldface), intergenic (lowercase), and initiation (boldface italics) nucleotides are underlined. The restriction sites of the multicloning site are indicated. The sequences of the ends of the HBsAg coding region (light gray arrow) and the 9 nucleotides added upstream or downstream of this coding region to comply with the rule of six (38) are indicated below the ATU sequence. (B) Time course of cell-associated (left) and cell-free (right) virus production in Vero/hSLAM cells infected with four recombinant viruses: MVvac2 (triangles), MVvac2(HBsAg)P (dots), MVvac2 (HBsAg)H (squares), and MVvac2(HBsAg)L (diamonds). Viral titers, indicated on the vertical axes, were measured 12, 24, 36, 48, 72, or 96 h postinfection. For clarity, symbols for viruses were moved slightly on the horizontal axes. Averages and standard deviations of three independent experiments are indicated.

Suppression of the antitetanus antibody response. One week after vaccination with MVvac2 or MVvac2(HBsAg)H, monkeys were immunized with tetanus toxoid at $20 \mu \mathrm{l} / \mathrm{kg}$ body weight s.c. (unconcentrated tetanus toxoid; Colorado Serum Company, Denver, CO). Tetanus antibody titers were determined at 21 days postvaccination. Titers of serum antibody were measured by ELISA using purified tetanus toxoid (Accurate Chemical and Scientific Corp., Westbury, NY) and positive/negative control rhesus sera as described previously (36).

Cell-mediated immunity. MV-specific T cells were counted using an gamma IFN (IFN- $\gamma$ ) enzyme-linked immunospot (ELISPOT) assay as previously de- scribed (36). Briefly, peripheral blood mononuclear cells (PBMC) were resuspended at $5 \times 10^{6}$ cells $/ \mathrm{ml}$ in a 48 -well flat-bottom plate in AIM V medium (Gibco/Invitrogen Corp., Grand Island, NY) supplemented with $10 \%$ fetal calf serum and stimulated overnight with live MV, Edmonston strain (American Type Culture Collection), at $10^{3} \mathrm{TCID}_{50} / 100 \mu \mathrm{l}$. Positive control stimulation was with $10 \mathrm{ng} / \mathrm{ml}$ phorbol 12-myristate 13 -acetate and $1 \mu \mathrm{g} / \mathrm{ml}$ ionomycin (Sigma, St. Louis, MO). Following overnight incubation, the cells were transferred to a 96-well ELISPOT plate coated with antibody to rhesus IFN- $\gamma$ (U-Cytech BV, Utrecht, The Netherlands) and developed as described by the manufacturer. 
Spot-forming cells (SFC) were counted under a dissecting microscope, and the numbers of spots in duplicate wells were averaged. A positive result was at least 10 spots per well and equal to or more than the mean plus 2 standard deviations of the medium control. The spot number in medium control wells was subtracted from the experimental spot count, and the number of SFC was adjusted to $10^{6}$ PBMC.

\section{RESULTS}

Generation of vectored MVs expressing HBsAg. We previously described an MV cDNA with vaccine-equivalent, but not vaccine-identical, coding capacity (11). Three coding differences between the polymerase (L) gene of this cDNA and those of vaccine strains Moraten and Schwartz $(32,33)$ exist. Although these three mutations concern nonconserved amino acids and although subsequent studies failed to reveal functional differences between viruses generated from the two genomic plasmids, it was considered appropriate to perform animal studies only with viruses having MV-identical coding capacity. Thus, the $\mathrm{pB}(+) \mathrm{MVvac} 2$ backbone (Fig. 1A) was constructed as detailed in Materials and Methods. We refer to viruses derived from this backbone as having a vaccine strain background.

To express HBsAg at different levels, we then added to $\mathrm{pB}(+) \mathrm{MVvac} 2$ an expression cassette (ATU). The ATU was inserted at three different positions: after the $\mathrm{P}$ gene (position 3373), after the $\mathrm{H}$ gene (position 9175), and after the $\mathrm{L}$ gene (position 15786). To direct HBsAg expression, the 681-nucleotide HBsAg (subtype ayw) coding region was then introduced in these plasmids to yield $\mathrm{pB}(+) \mathrm{MVvac} 2(\mathrm{HBs} \mathrm{Ag}) \mathrm{P}, \mathrm{pB}(+)$ MVvac2(HBsAg)H, and pB(+)MVvac2(HBsAg)L (Fig. 1A). The corresponding recombinant MVs were then generated.

To assess the replication efficiency of these vectored MVs, growth kinetics were determined. As shown in Fig. 1B, the maximum titers reached by all three vectored viruses were equivalent to those of the parental strain, MVvac2. Peak titers of about $10^{7} \mathrm{TCID}_{50} / \mathrm{ml}$ were reached $48 \mathrm{~h}$ postinfection for the cell-associated virus and of $3 \times 10^{5}$ to $10^{6} \mathrm{TCID}_{50} / \mathrm{ml} 48$ to $72 \mathrm{~h}$ postinfection for cell-free virus. Thus, the replication efficiency of these three HBsAg-vectored MVs was equivalent to that of the parental strain.

HBsAg expressed by vectored MVs is secreted in subviral particles. The HBsAg expressed from vectored MV was then characterized. Extracts of infected cells were assayed by immunoprecipitation using an anti-HBsAg rabbit polyclonal antiserum or an antiserum directed against the MV $\mathrm{H}$ glycoprotein. As shown in Fig. 2A, bottom, anti-HBsAg antibodies recognized the two differentially glycosylated $\mathrm{HBsAg}$ isoforms, with molecular masses of 26 and $24 \mathrm{kDa}$, respectively. As expected, MVvac2(HBsAg)P expressed HBsAg at the highest levels and MVvac2(HBsAg)L expressed HBsAg at the lowest (Fig. 2A, bottom, lanes $\mathrm{P}$ and $\mathrm{L}$, respectively). $\mathrm{H}$ protein was expressed at similar levels by all four viruses and was detected as a doublet (Fig. 2A, top; the upper band corresponds to the mannose-rich endoplasmic reticulum form and the lower band to the post-Golgi form with processed oligosaccharides) (7).

We also compared HBsAg expression levels by fluorescenceactivated cell sorting using $\mathrm{HBsAg}$ monoclonal antibodies (Fig. 2B) and MV N protein antibodies (Fig. 2B). The intensity of HBsAg-specific fluorescence was highest for MVvac2 (HBsAg)P-infected cells (mean = 286), intermediate for
A
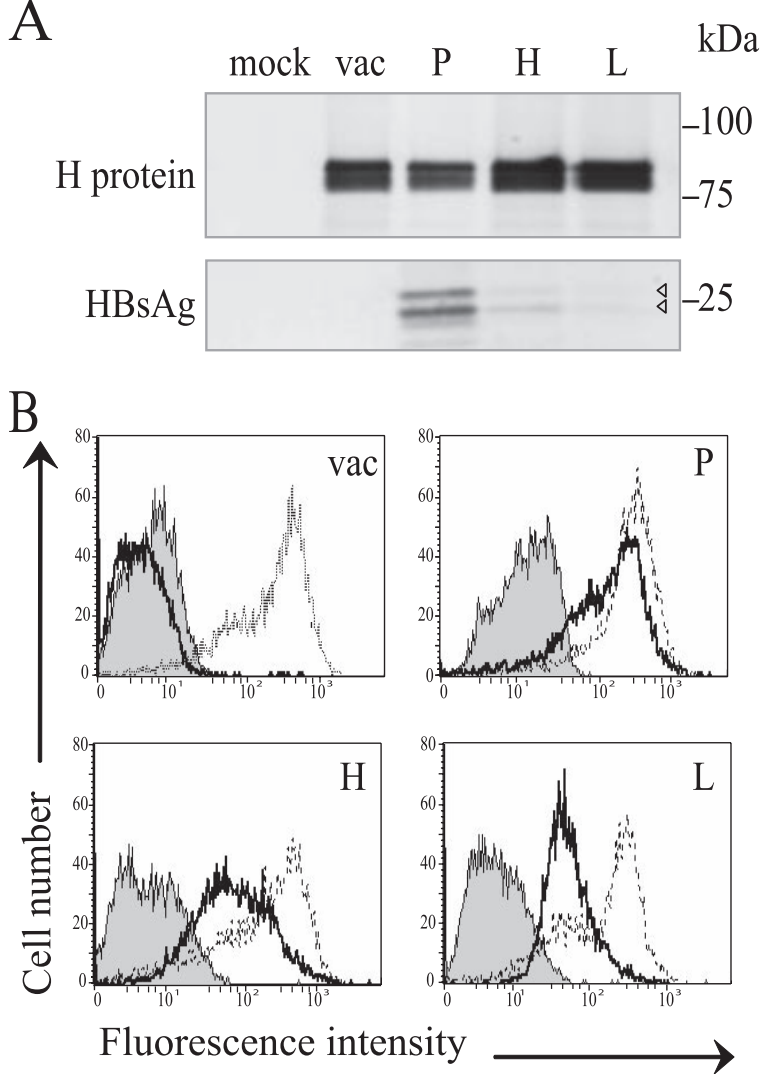

FIG. 2. HBsAg expression by vectored MV. (A) Immunoprecipitation of proteins produced by Vero/hSLAM cells infected with MVvac2 (vac), MVvac2(HBsAg)P (P), MVvac2(HBsAg)H (H), and MVvac2 (HBsAg)L (L). Mock, mock-infected cells. Proteins were labeled with $\left.{ }^{35} \mathrm{~S}\right]$ methionine 20 to $24 \mathrm{~h}$ postinfection and precipitated either with an H-specific serum (top) or an HBsAg-specific serum (bottom). The positions of molecular mass standards are indicated on the right. Arrowheads indicate two weak HBsAg signals. (B) Fluorescence-activated cell sorter analysis of HBsAg and MV N protein expression in infected cells. Thick lines, HBsAg expression; thin lines, $\mathrm{N}$ expression; shaded area, negative control without primary antibody.

MVvac2(HBsAg)H-infected cells (mean = 153), and lowest for MVvac2(HBsAg)L-infected cells (mean = 74). The signal levels of the $\mathrm{N}$ expression signal and of the negative controls (secondary antibody alone) were similar among cells infected with MVvac2 and its derivates. In particular, the $\mathrm{N}$ protein expression means were 508, 405, and 267 for cells infected with vectors expressing $\mathrm{HBs} A \mathrm{~g}$ from the $\mathrm{P}, \mathrm{H}$, and $\mathrm{L}$ positions, respectively. To quantitate $\mathrm{HBsAg}$ secretion, we used an ELISA as illustrated in Fig. 3A. We found that cells infected with MVvac2(HBsAg)P secreted up to $210 \mathrm{ng} / \mathrm{ml} \mathrm{HBsAg}$, whereas cells infected with MV expressing HBsAg from the two downstream positions secreted two- and four-times-lower HBsAg concentrations, respectively.

Most HBsAg in the serum of HBV-infected patients is in the form of spherical 22-nm particles devoid of nucleic acids, representing excess envelope material synthesized by HBV-infected cells (15). These subviral particles are also the principal constituent of the HBV vaccine. To assess whether HBsAg secreted by the vectored MV-infected cells associates in subviral particles, supernatants were clarified, concentrated, and 


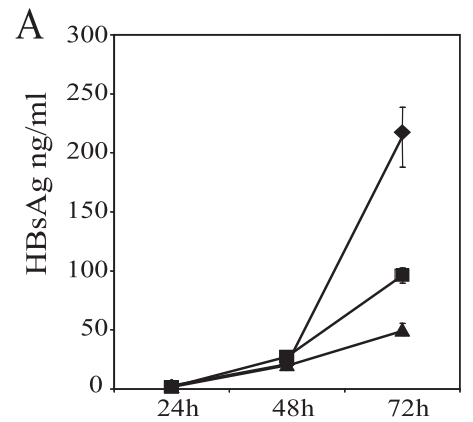

Time postinfection
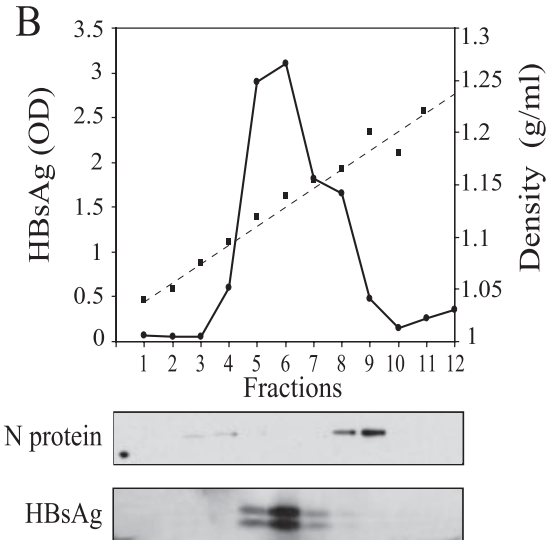

FIG. 3. Quantitation of HBsAg expression, and physical characteristics of released HBsAg. (A) Vero/hSLAM cells were infected with MVvac2(HBsAg)P (diamonds), MVvac2(HBsAg)H (squares), or $\mathrm{MVvac}$ (HBsAg)L (triangles), and media were collected at the time points indicated and clarified. HBsAg was assayed by ELISA and quantified by comparison with a standard curve. Averages and standard deviations of a triplicate experiment are shown. (B, top) Materials released from Vero/hSLAM cells infected with MVvac2(HBsAg)P. Media collected $72 \mathrm{~h}$ after infection were clarified, and virus was pelleted, loaded on a 20 to $60 \%$ sucrose gradient, and centrifuged to equilibrium. Onemilliliter fractions were collected from the top (left) to the bottom (right) and weighed. The density profile is shown by squares joined by a dashed line. The HBsAg concentration determined by ELISA is shown by dots joined by a continuous line. (Bottom) Aliquots of each fraction separated by $12.5 \%$ SDS-polyacrylamide gel electrophoresis, immunoblotted, and probed with the antibodies indicated on the left.

subjected to equilibrium sedimentation through a discontinuous sucrose step gradient. A density profile of such a gradient is shown in Fig. 3B. HBsAg concentration (Fig. 3B) peaked at 1.12 to $1.15 \mathrm{~g} / \mathrm{ml}$, corresponding to the density of subviral particles.

Additionally, samples from the sucrose gradient were probed using $\mathrm{HBsAg}$ and $\mathrm{MV} \mathrm{N}$ antibodies in immunoblots (Fig. 3B, bottom). The MV N protein was localized mainly in fractions 8 and 9, with a buoyant density of 1.16 to $1.18 \mathrm{mg} / \mathrm{ml}$, the expected density of MV particles (13), and thus physically separated from HBsAg (Fig. 3B, lower panels). This result indicates that most $\mathrm{HBsAg}$ is not incorporated in $\mathrm{MV}$ virions and instead is secreted in subviral particles.

HBsAg-vectored MVs elicit a robust immune response in transgenic mice. The efficiencies of vectored MVs in inducing humoral immune responses in Ifnar ${ }^{\mathrm{ko}}$-CD46Ge mice susceptible to $\mathrm{MV}$ infection were compared. These mice express the
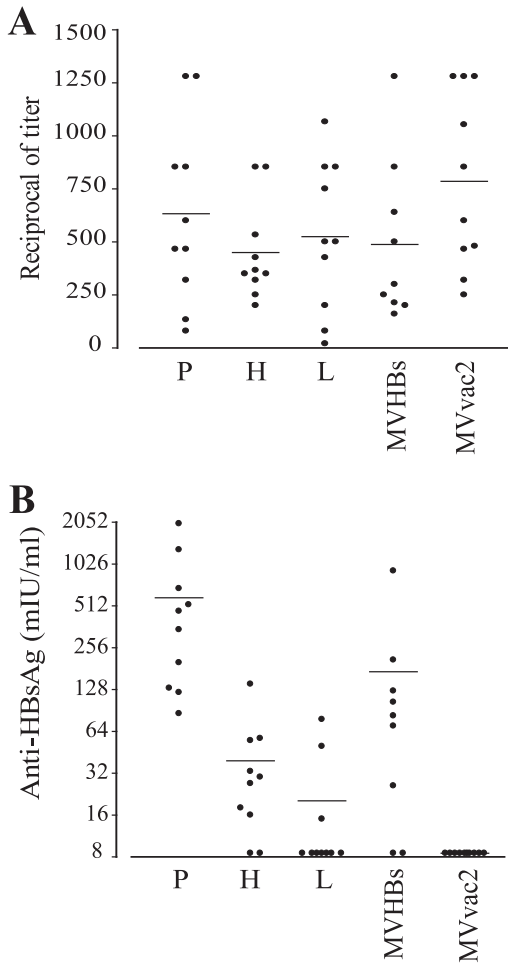

FIG. 4. Humoral immune response in genetically modified mice immunized with $\mathrm{HBsAg}$-expressing MV. Groups of 10 animals were immunized with MVvac2(HBsAg)P (P), MVvac2(HBsAg)H (H), MVvac2(HBsAg)L (L), MVHBs, or MVvac2. Sera obtained 28 days postimmunization were assayed for MV neutralization (A; reciprocals of the titer) or for anti-HBsAg levels (B; values from quantitative ELISA). Each dot represents an animal; the mean of the group is indicated by a horizontal bar.

MV vaccine strain receptor human CD46 with human-like tissue specificity in an IFN type I receptor knockout background (29). Groups of 10 mice were inoculated intraperitoneally with the three new HBsAg-expressing recombinants. In addition, two groups of mice were inoculated with MVHBs as a reference (43) or with the parental strain, MVvac2, as a negative control. The anti-MV neutralization titer and the anti-HBsAg response were assessed 28 days postinoculation. As shown in Fig. 4A, all animals showed average MV neutralization titers in the 1:400 to 1:800 range.

The anti-HBsAg response differed markedly between groups (Fig. 4B). The 10 mice immunized with MVvac2(HBsAg)P showed titers ranging from 80 to $2,000 \mathrm{mIU} / \mathrm{ml}$, with an average of $590 \mathrm{mIU} / \mathrm{ml}$. Eight of 10 mice inoculated with MVvac2 (HBsAg)H had positive responses with titers up to $128 \mathrm{mIU} / \mathrm{ml}$ and an average of $39 \mathrm{mIU} / \mathrm{ml}$. Only 3 of 10 mice immunized with MVvac2(HBsAg)L had an anti-HBsAg response with an average of $24 \mathrm{mIU} / \mathrm{ml}$, around twice the protection level in humans $(10 \mathrm{mIU} / \mathrm{ml})$. The anti-HBsAg response observed in MVHBs-immunized mice was on average $140 \mathrm{mIU} / \mathrm{ml}$, in line with a previous report (43). As expected none of the MVvac2immunized mice had a positive anti-HBsAg response. Thus, HBsAg expression from position $\mathrm{P}$ induced an anti-HBsAg response 15 times higher than the one induced by the virus that expresses HBsAg from position $\mathrm{H}$, which is remarkable be- 
TABLE 1. Rash and viremia in immunized Rhesus monkeys

\begin{tabular}{|c|c|c|c|c|c|}
\hline \multirow[b]{2}{*}{ Vaccine strain } & \multirow[b]{2}{*}{ Identification } & \multicolumn{2}{|c|}{ Immunization } & \multicolumn{2}{|c|}{ Challenge } \\
\hline & & Rash & $\begin{array}{c}\text { Viremia }^{a} \\
\text { (TCID }_{50} / \\
\left.10^{6} \mathrm{PBMC}^{2}\right)\end{array}$ & Rash & $\begin{array}{c}\text { Viremia }^{a} \\
{\left(\mathrm{TCID}_{50} /\right.} \\
\left.10^{6} \mathrm{PBMC}^{2}\right)\end{array}$ \\
\hline \multirow[t]{4}{*}{ MVvac2 } & 336 & No & 0 & No & 0 \\
\hline & 156 & No & 0 & No & 0 \\
\hline & 157 & No & $10^{0}-10^{0.75}$ & No & $10^{0.75}$ \\
\hline & 286 & No & $10^{0}-10^{0.75}$ & No & 0 \\
\hline \multirow[t]{4}{*}{ MVvac2(HBsAg)P } & 547 & No & 0 & No & 0 \\
\hline & 676 & No & $10^{0}-10^{0.75}$ & No & 0 \\
\hline & 683 & No & 0 & No & 0 \\
\hline & 782 & No & 0 & No & 0 \\
\hline \multirow[t]{4}{*}{ MVvac2(HBsAg)H } & 380 & No & 0 & No & 0 \\
\hline & 300 & No & 0 & No & 0 \\
\hline & 360 & No & 0 & No & 0 \\
\hline & 397 & No & 0 & No & $10^{1}$ \\
\hline \multirow[t]{2}{*}{ Moraten (Attenuvax) } & 173 & No & 0 & No & $10^{0.75}$ \\
\hline & 221 & No & 0 & No & 0 \\
\hline \multirow{2}{*}{ None (naive) } & & $\mathrm{NA}^{b}$ & NA & Yes & $10^{3.25}$ \\
\hline & 301 & NA & NA & Yes & $10^{3.75}$ \\
\hline
\end{tabular}

${ }^{a}$ On day 7 postimmunization or postchallenge.

${ }^{b} \mathrm{NA}$, not applicable.

cause HBsAg expression levels differ only by a factor of about 2. Another twofold HBsAg expression level decrease in MVvac2(HBsAg)L also interfered strongly with HBsAg immunogenicity. To assess whether such effects occurred in another animal system, we inoculated macaques with the two viruses expressing HBsAg at the highest levels.

Vectored MV protects rhesus macaques from MV challenge. To assess whether the vectored MV retained vaccine competence, groups of four rhesus macaques were immunized by the s.c. route with MVvac2 or its derivatives expressing $\mathrm{HBsAg}$ from position $\mathrm{P}$ or $\mathrm{H}$. As controls, two animals were immunized with an equivalent dose of the vaccine Moraten strain. Two other monkeys were not vaccinated, for a total of 16 experimental animals that were all challenged 6 to 10 weeks after vaccination with $10^{5}$ TCID $_{50}$ of MV Davis My $_{87}$ by the conjuctival/intranasal route (26). To compare levels of protective efficacy and the induction of host responses, three quantitative parameters were measured: levels of viremia, anti-MV neutralization titers, and number of MV-specific IFN- $\gamma$-secreting T cells. These parameters were assayed before and after vaccination and challenge. In addition, rash appearance was scored.

Table 1 documents measles-specific rash appearance and levels of viremia. None of the animals developed a measlesspecific skin rash at any time after vaccination. Viremia was assayed in PBMC samples collected 4, 7, and 14 days after immunization and challenge. Virus was isolated from two animals inoculated with MVvac2 and from one animal inoculated with MVvac2(HBsAg)P 7 days after inoculation. Low-level viremia, at $\leq 5 \mathrm{TCID}_{50}$ per $10^{6} \mathrm{PBMC}$, occurs after MV vaccination $(47,50)$. There was no rash or evidence of MV-induced suppression of the antibody response to tetanus immunization in all eight monkeys vaccinated with either MVvac2 or MVvac2(HBsAg)H in which the antitetanus antibody response was assayed (data not shown). In contrast, the two control naive animals presented a classic measles rash 7 days after challenge and levels of viremia of $10^{3}$ to $10^{4} \mathrm{TCID}_{50}$ per $10^{6}$ PBMC, characteristic of pathogenic MV infection (47, 51). One animal in three of the four vaccine groups had viremia, but at levels of $10 \mathrm{TCID}_{50}$ or less per $10^{6} \mathrm{PBMC}$.

Figure 5A documents the MV neutralization titers 28 days after immunization or 28 days after challenge. Neutralization titers from 1:100 to 1:420 were monitored in response to vaccination. These titers increased by a factor of 3 to 10 in response to the challenge. The two naive monkeys developed neutralization titers of 1:200 and 1:640 28 days postchallenge, in line with previous reports $(46,47,51)$.

To evaluate the cell-mediated immune (CMI) response, we isolated MV-specific IFN- $\gamma$-secreting T cells by ELISPOT at 0 and 30 days after vaccination and challenge (Table 2). Thirty days postvaccination, most of the animals immunized with recombinant $\mathrm{MV}$ presented a CMI response (group averages of $63 \mathrm{SFC} / 10^{6} \mathrm{PBMC}$ for MVvac2 and 44 and $55 \mathrm{SFC} / 10^{6} \mathrm{PBMC}$
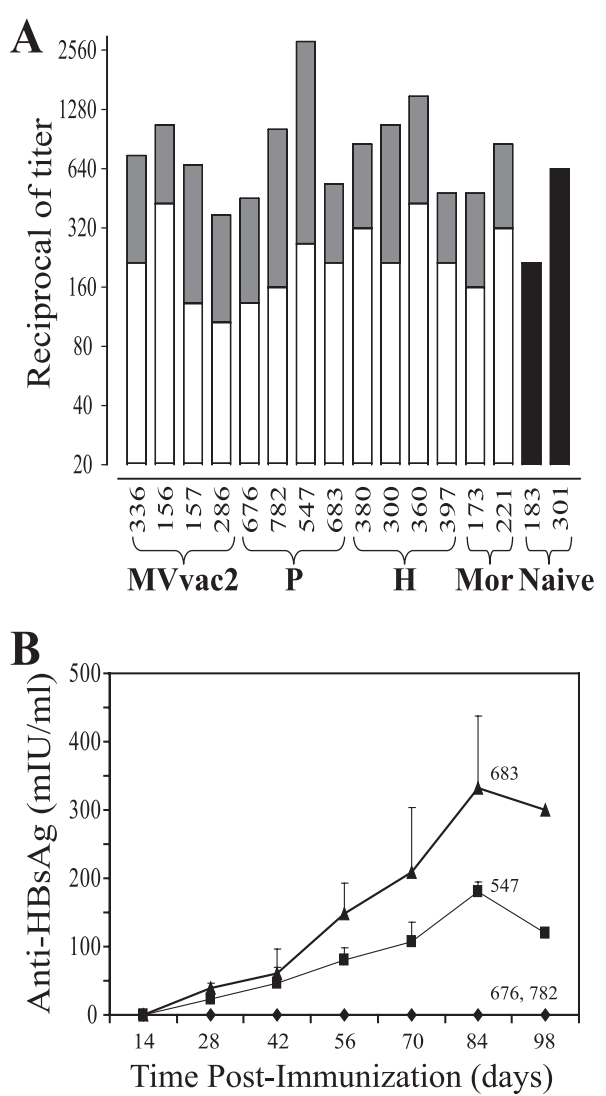

FIG. 5. Humoral immune response in rhesus monkeys immunized with HBsAg-expressing MV. (A) Monkeys were immunized with the vectored MV indicated at the bottom. P, MVvac2(HBsAg)P; H, $\mathrm{MVvac} 2(\mathrm{HBsAg}) \mathrm{H}$. Mor, monkeys immunized with a dose of Moraten vaccine. Naive, control animals that remained naive before challenge. Anti-MV neutralization titers measured 28 days postimmunization are indicated by the heights of the open bars, and the increase in titer measured 28 days postchallenge are indicated by the heights of the solid gray bars. Black bars, titers measured 28 days after challenge of naive monkeys. (B) Time course of the anti-HBsAg immune response in MVvac2(HBsAg)P-inoculated rhesus monkeys. The rhesus monkey identification number is shown above each curve. Averages and standard deviations of at least three independent determinations are shown. 
TABLE 2. MV-specific IFN- $\gamma$ ELISPOT

\begin{tabular}{|c|c|c|c|c|c|}
\hline \multirow{3}{*}{ Vaccine strain } & \multirow{3}{*}{ Identification } & \multicolumn{4}{|c|}{$\begin{array}{l}\text { CMI response }{ }^{a}\left(\mathrm{SFC} / 10^{6}\right. \\
\text { PBMC) for: }\end{array}$} \\
\hline & & \multicolumn{2}{|c|}{$\begin{array}{l}\text { Immunization } \\
\text { on day: }\end{array}$} & \multicolumn{2}{|c|}{$\begin{array}{l}\text { Challenge } \\
\text { on day: }\end{array}$} \\
\hline & & 0 & 30 & 0 & 30 \\
\hline \multirow[t]{4}{*}{ MVvac2 } & 336 & 0 & 16 & 0 & 29 \\
\hline & 156 & $\mathrm{ND}^{b}$ & 85 & 108 & 111 \\
\hline & 157 & 75 & 9 & ND & 83 \\
\hline & 286 & 0 & 41 & ND & 21.5 \\
\hline \multirow[t]{4}{*}{ MVvac2(HBsAg)P } & 547 & 0 & 36 & 38 & 148 \\
\hline & 676 & 0 & 28 & 24 & 6 \\
\hline & 683 & 7 & 74 & 28 & 67 \\
\hline & 782 & 0 & 41 & 40 & 34 \\
\hline \multirow[t]{4}{*}{ MVvac2(HBsAg)H } & 380 & 0 & 18 & 60 & 58 \\
\hline & 300 & 27 & 57 & 54 & 150 \\
\hline & 360 & 14 & 71 & 84 & 32 \\
\hline & 397 & 0 & 38 & 70 & 45 \\
\hline \multirow[t]{2}{*}{ Moraten (Attenuvax) } & 173 & 5 & 136 & 139 & 161 \\
\hline & 221 & ND & 9 & 61 & 40 \\
\hline \multirow[t]{2}{*}{ None (naive) } & 183 & $\mathrm{NA}^{c}$ & NA & 15 & 32 \\
\hline & 301 & NA & NA & 0 & 76 \\
\hline
\end{tabular}

${ }^{a}$ Positive results (see Materials and Methods) are in boldface.

${ }^{b} \mathrm{ND}$, not determined.

${ }^{c}$ NA, not applicable.

for the P- and H-vectored HBsAg MV). This response was maintained until the day of challenge. Thirty days after challenge an increase in CMI response was documented in one animal per group. Overall, the CMI response observed in animals inoculated with vectored $\mathrm{MV}$ was equivalent to that from the Moraten (Attenuvax)-vaccinated animals and did not reveal differences between the groups. One monkey (no. 300) had detectable MV-specific T cells prior to vaccination at 7 SFC above the cutoff, which was unexpected.

HBsAg humoral response in macaques. To assess the humoral immune response against $\mathrm{HBsAg}$, antibody levels were measured by ELISA. Two of four monkeys immunized with MVvac2(HBsAg)P and no monkey vaccinated with MVvac2 (HBsAg)H had an anti-HBsAg response. Figure 5B documents the time course of the HBsAg response in MVvac2(HBsAg)Pimmunized monkeys: low levels 4 weeks postimmunization and a steady increase until week 12 , reaching peak titers of 180 and $340 \mathrm{mIU} / \mathrm{ml}$, or 18 to 34 times the reported protective level for humans. In summary, MVvac2 and its vectored derivatives elicited equivalent protection from pathogenic MV challenge as the standard vaccine. Only the vectored virus expressing HBsAg at the highest levels induced a strong immune response to HBsAg in two of four macaques.

\section{DISCUSSION}

We show here that vectored MVs expressing HBsAg are as effective as the live attenuated MV vaccine in protecting macaques from challenge with a pathogenic MV strain. Our study also revealed that only the MV expressing HBsAg at the highest levels elicited a strong humoral HBsAg response in some macaques. Similarly, in a rodent model HBsAg expression levels determined the strength of the humoral immune response but a lower expression level elicited a strong immune response. These observations have implications for the development of divalent vaccines based on MV and other live attenuated viruses.

Recombinant MVs with additional vaccine specificities have been generated and their immunogenicities tested in animal models $(10,24,43,50,52)$. However, for none of these viruses was formal proof of retention of vaccine efficacy sought, as done here through challenge of vaccinated hosts with a macaque-adapted pathogenic MV strain. It appears likely that recombinant MVs with vaccine-identical coding sequences retain vaccine efficacy $(8,10,24)$. However, in the original MV infectious cDNA (37) mutations interfering with the innate immunity control function of both $\mathrm{V}$ and $\mathrm{P}$ proteins exist (11, $30)$. Thus, recombinant viruses derived from it $(43,50,52)$ may be overattenuated. We did not measure significant differences between the immunogenicity of the MVHBs vector (43) based on the original MV genomic cDNA and those of the new HBsAg-expressing viruses generated here. However, the IFNdefective mice we inoculated are not appropriate for investigating the effects of defects in viral proteins controlling innate immunity. On the other hand, another comparative analysis of the immunogenicities of MV derived from the original infectious cDNA and the Schwartz vaccine strain did suggest overattenuation (8).

The vast difference in the strength of the HBsAg-specific humoral immune response elicited from vectored MVs that express this protein at levels differing by a factor of 2 is remarkable. In macaques, only the vector expressing HBsAg at the highest level induced a detectable humoral response. In mice, this vector was strongly immunogenic; the vector expressing one-half as much HBsAg induced 10- to 20-timeslower levels of HBsAg antibodies, and the vector expressing about four times less HBsAg was weakly immunogenic in 3 of 10 animals. Thus, small HBsAg expression differences do have striking effects on the magnitude of humoral immune response induction in two different hosts.

The strength of the immune response may be strikingly concentration dependent also for antigens that, unlike $\mathrm{HBsAg}$, do not form virus-like particles. A review of published studies on vectored MV indicates that most of the successful immunity induction strategies relied on $\mathrm{P}$ position expression, whereas the immune response to foreign proteins expressed from the ATU inserted at the $\mathrm{H}$ position was rarely documented (10, 24, $43,50,52)$. Other negative-strand RNA viruses with nonsegmented genomes have also been developed as vaccine vectors $(2,48)$. Several of the successful experimental vaccines are based on envelope protein exchange rather than on addition of the foreign envelope protein $(3,14,19)$, but a bovine/human parainfluenza virus 3 expressing the human respiratory syncytial virus $\mathrm{F}$ glycoprotein in addition to the vector proteins has reached the clinical trial stage. This recombinant virus expresses the foreign antigen from upstream of $\mathrm{N}$ and therefore at the highest levels, another indication that high expression levels are important (41).

We did generate an MV expressing HBsAg from an ATU located upstream of $\mathrm{N}$, but this virus reached titers of only $10^{5}$ $\mathrm{TCID}_{50} / \mathrm{ml}$ (data not shown), which is 100 times lower than those of the other HBsAg-expressing viruses, suggesting inter- 
ference with virus growth. We have expressed several other proteins from a location upstream of $\mathrm{N}$; in this location, transcription initiates in the original position of $\mathrm{N}$ transcription initiation, conserving a hexameric phase supportive of high expression (23). Expression of most proteins at high levels did not result in interference effects, but two vectors had issues. First, a recombinant MV expressing the sodium-iodine symporter from upstream of $\mathrm{N}$ grew to low titers, whereas viruses expressing it from downstream of $\mathrm{P}$ or $\mathrm{H}$ grew to normal titers (12; D. Dingli and R. Cattaneo, unpublished observations). Second, a recombinant canine distemper virus expressing the green fluorescent protein from upstream of $\mathrm{N}$ was attenuated in ferrets, whereas viruses expressing it from downstream of $\mathrm{P}$ or $\mathrm{H}$ were fully virulent (49). Thus, high levels of expression of certain proteins, including $\mathrm{HBsAg}$, can interfere with viral growth in cultivated cells or with spread in a natural host. On the other hand, we show here that strong HBsAg expression is necessary to elicit humoral immunity. Thus, for vaccination purposes proteins should be expressed at the highest levels compatible with efficient viral replication.

Expression of HBsAg from the L position was only 4 to 5 times lower than that from the $\mathrm{P}$ position, whereas in standard infections 10 to 20 times more $\mathrm{P}$ than $\mathrm{L}$ mRNAs are detected $(6,35)$. Thus, the ratio of HBsAg expression between the $\mathrm{P}$ and L positions was two to three times higher than expected. Several possibilities may account for this, including posttranscriptional effects or subtle differences in replication. Interestingly, initiation of transcription of the ATUs in the $\mathrm{P}$ and $\mathrm{H}$ positions was in the 0 hexameric phase, whereas initiation of transcription of the ATU in the L position is in the +5 hexameric phase.

It is possible that even the two macaques with negative HBsAg antibody counts after inoculation with MVvac2 (HBsAg)P did develop HBV immunity. Chimpanzees inoculated with $\mathrm{HBs} A g$-vectored vaccinia viruses or adenoviruses did not develop clinical hepatitis after HBV challenge, even when HBsAg antibodies had not been detected $(25,27)$. Moreover, a second dose of $\mathrm{MVvac} 2(\mathrm{HBsAg}) \mathrm{P}$ may raise the HBsAg seroconversion rate. Alternatively, one dose of the protein-based HBV vaccine may be sufficient to elicit strong immunity in individuals primed with $\mathrm{MVvac} 2(\mathrm{HBs} \mathrm{Ag}) \mathrm{P}$ that did not seroconvert. Finally, HBV immunity may be boosted through inoculation of other HBsAg-expressing vectors. For example, inoculation of children in need of variola virus or adenovirus immunization with $\mathrm{HBsAg-vectored} \mathrm{vaccinia} \mathrm{vi-}$ ruses or adenoviruses may boost $\mathrm{HBV}$ immunity originally conferred by the MV-HBV vaccine. Thus, divalent or multivalent vaccines based on viruses from different families may be developed to offer appropriate vaccination solutions for the different necessities of populations or individuals.

\section{ACKNOWLEDGMENTS}

We thank Heinz Schaller and Christa Kuhn for antibodies; Joseph Yao, Christoph Springfeld, and Chanakha Navaratnarajah for help and for reading the manuscript; Marie Frenzke and Sompong Vongpunsawad for excellent technical support; and Hannah Koble for secretarial assistance.

The National Institutes of Health (AI57761) and the Mayo Foundation supported this research. J.R.V. is a scholar of the National Researcher System, CONACyT, and IPN (Mexico).

\section{REFERENCES}

1. Boland, G., J. Beran, M. Lievens, J. Sasadeusz, P. Dentico, H. Nothdurft, J. N. Zuckerman, B. Genton, R. Steffen, L. Loutan, J. Van Hattum, and M. Stoffel. 2004. Safety and immunogenicity profile of an experimental hepatitis B vaccine adjuvanted with AS04. Vaccine 23:316-320.

2. Bukreyev, A., M. H. Skiadopoulos, B. R. Murphy, and P. L. Collins. 2006 Nonsegmented negative-strand viruses as vaccine vectors. J. Virol. 80:1029310306.

3. Bukreyev, A., L. Yang, S. R. Zaki, W. J. Shieh, P. E. Rollin, B. R. Murphy, P. L. Collins, and A. Sanchez. 2006. A single intranasal inoculation with a paramyxovirus-vectored vaccine protects guinea pigs against a lethal-dose Ebola virus challenge. J. Virol. 80:2267-2279.

4. Cassidy, W. M., B. Watson, V. A. Ioli, K. Williams, S. Bird, and D. J. West. 2001. A randomized trial of alternative two- and three-dose hepatitis B vaccination regimens in adolescents: antibody responses, safety, and immunologic memory. Pediatrics 107:626-631.

5. Cathomen, T., H. Y. Naim, and R. Cattaneo. 1998. Measles viruses with altered envelope protein cytoplasmic tails gain cell fusion competence. J. Virol. 72:1224-1234.

6. Cattaneo, R., G. Rebmann, A. Schmid, K. Baczko, V. ter Meulen, and M. A. Billeter. 1987. Altered transcription of a defective measles virus genome derived from a diseased human brain. EMBO J. 6:681-688.

7. Cattaneo, R., and J. K. Rose. 1993. Cell fusion by the envelope glycoproteins of persistent measles viruses which caused lethal human brain disease. J. Virol. 67:1493-1502.

8. Combredet, C., V. Labrousse, L. Mollet, C. Lorin, F. Delebecque, B. Hurtrel, H. McClure, M. B. Feinberg, M. Brahic, and F. Tangy. 2003. A molecularly cloned Schwarz strain of measles virus vaccine induces strong immune responses in macaques and transgenic mice. J. Virol. 77:11546-11554.

9. Cutts, F. T., and L. E. Markowitz. 1994. Successes and failures in measles control. J. Infect. Dis. 170(Suppl. 1):S32-S41.

10. Despres, P., C. Combredet, M. P. Frenkiel, C. Lorin, M. Brahic, and F. Tangy. 2005. Live measles vaccine expressing the secreted form of the West Nile virus envelope glycoprotein protects against West Nile virus encephalitis. J. Infect. Dis. 191:207-214.

11. Devaux, P., V. von Mesling, W. Songsungthong, C. Springfeld, and R. Cattaneo. 2007. Tyrosine 110 in the measles virus phosphoprotein is required to block STAT 1 phosphorylation. Virology 360:72-83.

12. Dingli, D., K. W. Peng, M. E. Harvey, P. R. Greipp, M. K. O'Connor, R. Cattaneo, J. C. Morris, and S. J. Russell. 2004. Image-guided radiovirotherapy for multiple myeloma using a recombinant measles virus expressing the thyroidal sodium iodide symporter. Blood 103:1641-1646.

13. Galinski, M. S., and S. L. Wechsler. 1991. The molecular biology of the the paramyxovirus genus, p. 41-82. In D. W. Kingsbury (ed.), The paramyxoviruses. Plenum Press, New York, NY.

14. Geisbert, T. W., S. Jones, E. A. Fritz, A. C. Shurtleff, J. B. Geisbert, R. Liebscher, A. Grolla, U. Stroher, L. Fernando, K. M. Daddario, M. C. Guttieri, B. R. Mothe, T. Larsen, L. E. Hensley, P. B. Jahrling, and H. Feldmann. 2005. Development of a new vaccine for the prevention of Lassa fever. PLoS Med. 2:e183.

15. Gerin, J. L., R. H. Purcell, M. D. Hoggan, P. V. Holland, and R. M. Chanock. 1969. Biophysical properties of Australia antigen. J. Virol. 4:763-768.

16. Hilleman, M. R. 2001. Current overview of the pathogenesis and prophylaxis of measles with focus on practical implications. Vaccine 20:651-665.

17. Hinman, A. 1999. Eradication of vaccine-preventable diseases. Annu. Rev. Public Health 20:211-229.

18. Iverson, L. E., and J. K. Rose. 1981. Localized attenuation and discontinuous synthesis during vesicular stomatitis virus transcription. Cell 23:477-484.

19. Jones, S. M., H. Feldmann, U. Stroher, J. B. Geisbert, L. Fernando, A. Grolla, H. D. Klenk, N. J. Sullivan, V. E. Volchkov, E. A. Fritz, K. M. Daddario, L. E. Hensley, P. B. Jahrling, and T. W. Geisbert. 2005. Live attenuated recombinant vaccine protects nonhuman primates against Ebola and Marburg viruses. Nat. Med. 11:786-790.

20. Kamiyama, T., H. Sato, T. Takahara, S. Kageyama, and K. Shiraki. 2000. Novel immunogenicity of Oka varicella vaccine vector expressing hepatitis B surface antigen. J. Infect. Dis. 181:1158-1161.

21. Karber, G. 1931. Beitrag zur kollektiven Behandlung pharmakologischer Reihenversuche. Arch. Exp. Pathol. Pharmakol. 162:480-483.

22. Keating, G. M., and S. Noble. 2003. Recombinant hepatitis B vaccine (Engerix-B): a review of its immunogenicity and protective efficacy against hepatitis B. Drugs 63:1021-1051.

23. Kolakofsky, D., T. Pelet, D. Garcin, S. Hausmann, J. Curran, and L. Roux. 1998. Paramyxovirus RNA synthesis and the requirement for hexamer genome length: the rule of six revisited. J. Virol. 72:891-899.

24. Lorin, C., L. Mollet, F. Delebecque, C. Combredet, B. Hurtrel, P. Charneau, M. Brahic, and F. Tangy. 2004. A single injection of recombinant measles virus vaccines expressing human immunodeficiency virus (HIV) type 1 clade B envelope glycoproteins induces neutralizing antibodies and cellular immune responses to HIV. J. Virol. 78:146-157.

25. Lubeck, M. D., A. R. Davis, M. Chengalvala, R. J. Natuk, J. E. Morin, K. Molnar-Kimber, B. B. Mason, B. M. Bhat, S. Mizutani, P. P. Hung, et al. 
1989. Immunogenicity and efficacy testing in chimpanzees of an oral hepatitis $B$ vaccine based on live recombinant adenovirus. Proc. Natl. Acad. Sci. USA 86:6763-6767.

26. McChesney, M. B., C. J. Miller, P. A. Rota, Y. D. Zhu, L. Antipa, N. W. Lerche, R. Ahmed, and W. J. Bellini. 1997. Experimental measles. I. Pathogenesis in the normal and the immunized host. Virology 233:74-84.

27. Moss, B., G. L. Smith, J. L. Gerin, and R. H. Purcell. 1984. Live recombinant vaccinia virus protects chimpanzees against hepatitis B. Nature 311:67-69.

28. Moszynski, P. 2007. Measles campaign's "historic victory" for global public health. Br. Med. J. 334:177.

29. Mrkic, B., J. Pavlovic, T. Rulicke, P. Volpe, C. J. Buchholz, D. Hourcade, J. P. Atkinson, A. Aguzzi, and R. Cattaneo. 1998. Measles virus spread and pathogenesis in genetically modified mice. J. Virol. 72:7420-7427.

30. Ohno, S., N. Ono, M. Takeda, K. Takeuchi, and Y. Yanagi. 2004. Dissection of measles virus $\mathrm{V}$ protein in relation to its ability to block alpha/beta interferon signal transduction. J. Gen. Virol. 85:2991-2999.

31. Ono, N., H. Tatsuo, Y. Hidaka, T. Aoki, H. Minagawa, and Y. Yanagi. 2001 Measles viruses on throat swabs from measles patients use signaling lymphocytic activation molecule (CDw150) but not CD46 as a cellular receptor. J. Virol. 75:4399-4401.

32. Parks, C. L., R. A. Lerch, P. Walpita, H. P. Wang, M. S. Sidhu, and S. A Udem. 2001. Analysis of the noncoding regions of measles virus strains in the Edmonston vaccine lineage. J. Virol. 75:921-933.

33. Parks, C. L., R. A. Lerch, P. Walpita, H. P. Wang, M. S. Sidhu, and S. A Udem. 2001. Comparison of predicted amino acid sequences of measles virus strains in the Edmonston vaccine lineage. J. Virol. 75:910-920.

34. Pless, R. P., A. D. Bentsi-Enchill, and P. Duclos. 2003. Monitoring vaccine safety during measles mass immunization campaigns: clinical and programmatic issues. J. Infect. Dis. 187(Suppl. 1):S291-S298.

35. Plumet, S., W. P. Duprex, and D. Gerlier. 2005. Dynamics of viral RNA synthesis during measles virus infection. J. Virol. 79:6900-6908.

36. Premenko-Lanier, M., P. A. Rota, G. H. Rhodes, W. J. Bellini, and M. B. McChesney. 2004. Protection against challenge with measles virus (MV) in infant macaques by an MV DNA vaccine administered in the presence of neutralizing antibody. J. Infect. Dis. 189:2064-2071.

37. Radecke, F., P. Spielhofer, H. Schneider, K. Kaelin, M. Huber, C. Dotsch, G. Christiansen, and M. A. Billeter. 1995. Rescue of measles viruses from cloned DNA. EMBO J. 14:5773-5784.

38. Rager, M., S. Vongpunsawad, W. P. Duprex, and R. Cattaneo. 2002 Polyploid measles virus with hexameric genome length. EMBO J. 21:23642372 .

39. Reed, L. J., and H. Muench. 1938. A simple method of estimating fifty per cent endpoints. Am. J. Epidemiol. 27:493-497.

40. Richardson, C. D., A. Scheid, and P. W. Choppin. 1980. Specific inhibition of paramyxovirus and myxovirus replication by oligopeptides with amino acid sequences similar to those at the N-termini of the F1 or HA2 viral polypeptides. Virology 105:205-222.

41. Schmidt, A. C., D. R. Wenzke, J. M. McAuliffe, M. St Claire, W. R. Elkins, B. R. Murphy, and P. L. Collins. 2002. Mucosal immunization of rhesus monkeys against respiratory syncytial virus subgroups $\mathrm{A}$ and $\mathrm{B}$ and human parainfluenza virus type 3 by using a live cDNA-derived vaccine based on a host range-attenuated bovine parainfluenza virus type 3 vector backbone. J. Virol. 76:1089-1099.

42. Shiraki, K., Y. Hayakawa, H. Mori, J. Namazue, A. Takamizawa, I. Yoshida, K. Yamanishi, and M. Takahashi. 1991. Development of immunogenic recombinant Oka varicella vaccine expressing hepatitis B virus surface antigen. J. Gen. Virol. 72:1393-1399.

43. Singh, M., R. Cattaneo, and M. A. Billeter. 1999. A recombinant measles virus expressing hepatitis B virus surface antigen induces humoral immune responses in genetically modified mice. J. Virol. 73:4823-4828.

44. Smith, G. L., M. Mackett, and B. Moss. 1983. Infectious vaccinia virus recombinants that express hepatitis B virus surface antigen. Nature 302:490495.

45. Strebel, P. M., M. J. Papania, and N. A. Halsey. 2004. Measles vaccine, p. 389-440. In S. A. Plotkin and W. A. Orenstein (ed.), Vaccines, 4th ed. Saunders-Elsevier, Philadelphia, PA

46. van Binnendijk, R. S., M. C. Poelen, G. van Amerongen, P. de Vries, and A. D. Osterhaus. 1997. Protective immunity in macaques vaccinated with live attenuated, recombinant, and subunit measles vaccines in the presence of passively acquired antibodies. J. Infect. Dis. 175:524-532.

47. van Binnendijk, R. S., R. W. van der Heijden, G. van Amerongen, F. G. UytdeHaag, and A. D. Osterhaus. 1994. Viral replication and development of specific immunity in macaques after infection with different measles virus strains. J. Infect. Dis. 170:443-448.

48. von Messling, V., and R. Cattaneo. 2004. Toward novel vaccines and therapies based on negative strand RNA viruses. Curr. Top. Microbiol. Immunol. 283:281-312.

49. von Messling, V., D. Milosevic, and R. Cattaneo. 2004. Tropism illuminated: lymphocyte-based pathways blazed by lethal morbillivirus through the host immune system. Proc. Natl. Acad. Sci. USA 101:14216-14221.

50. Wang, Z., L. Hangartner, T. I. Cornu, L. R. Martin, A. Zuniga, M. A. Billeter, and H. Y. Naim. 2001. Recombinant measles viruses expressing heterologous antigens of mumps and simian immunodeficiency viruses. Vaccine 19:2329-2336.

51. Zhu, Y. D., J. Heath, J. Collins, T. Greene, L. Antipa, P. Rota, W. Bellini, and M. McChesney. 1997. Experimental measles. II. Infection and immunity in the rhesus macaque. Virology 233:85-92.

52. Zuniga, A., Z. Wang, M. Liniger, L. Hangartner, M. Caballero, J. Pavlovic, P. Wild, J. F. Viret, R. Glueck, M. A. Billeter, and H. Y. Naim. 2007. Attenuated measles virus as a vaccine vector. Vaccine 25:2974-2983. 